\title{
Cough peak flow to predict the extubation outcome: Comparison between three cough stimulation methods
}

\author{
Christiane Melo Almeida, $\mathrm{MSc}^{1}$, Agnaldo José Lopes, $\mathrm{PhD}^{1,2}$, Fernando Silva Guimarães, $\mathrm{PhD}^{3}$
}

CM Almeida, AJ Lopes, FS Guimarães. Cough peak flow to predict the extubation outcome: Comparison between three cough stimulation methods. Can J Respir Ther 2020;56:58-64. doi: 10.29390/cjrt-2020-037.

\begin{abstract}
Objectives: The purpose of this study was to compare the predictive value of three cough peak flow (CPF) maneuvers in predicting the extubation outcome in a cohort of mechanically ventilated subjects.

Methods: Eighty-one mechanically ventilated subjects who succeeded in the spontaneous breathing trial were included. In a randomized order, CPF was stimulated and measured using three methods: voluntary command (V_CPF), tracheal saline instillation (S_CPF), and mechanical stimulation with a catheter (C_CPF). Additionally, CPF was measured 20 min after the extubation (PE_CPF). The diagnostic accuracy of the CPF methods in relation to the extubation outcome was measured using the receiver operating characteristic (ROC) curve. ROC curve results were compared using the Hanley and McNeil method.

Results: The three methods presented high accuracy in predicting the extubation outcome $\left(V_{-} C P F=0.89, S_{-} C P F=0.93\right.$, and C_CPF $\left.=0.90\right)$, without statistically significant differences between them (V_CPF vs. S_CPF, $p=0.14 ; V_{-}$CPF vs. C_CPF, $p=0.84$; S_CPF vs. C_CPF, $\left.p=0.13\right)$. The optimum cutoff values were V_CPF $=45 \mathrm{~L} / \mathrm{min}, \mathrm{S}_{-} \mathrm{CPF}=60 \mathrm{~L} / \mathrm{min}$, and $\mathrm{C}_{-} \mathrm{CPF}=55 \mathrm{~L} / \mathrm{min}$. PE_CPF also showed high accuracy in predicting the extubation outcome $(\mathrm{AUC}=0.95$; cutoff $=75 \mathrm{~L} / \mathrm{min})$.

Conclusions: In mechanically ventilated and cooperative subjects, there is no difference in the accuracy of CPF measured voluntarily, with stimulation using saline or by catheter stimulation in predicting the reintubation. CPF recording after endotracheal tube removal has high accuracy to predict the extubation outcome.
\end{abstract}

Key Words: cough; peak expiratory flow rate; airway extubation; ventilator weaning; mechanical ventilation; risk assessment

\section{INTRODUCTION}

Mechanical ventilation (MV) is associated with significant adverse consequences, which worsen over time. Unnecessary delays in the weaning and extubation process can lead to complications such as ventilatorassociated pneumonia, ventilator-induced lung injury, atelectasis, pneumothorax, stress gastropathy, arrhythmias, volume retention, and malnutrition [1]. Conversely, if MV discontinuation is performed prematurely, this can lead to reintubation, which is associated with increased morbidity and mortality [2]. Therefore, the accurate assessment of subjects' readiness for weaning and extubation is of great clinical interest. The most recommended method for assessing the capacity of subjects to sustain spontaneous ventilation is a 30 - or 120 -min spontaneous breathing trial (SBT) [3]. However, even after passing the SBT, the reintubation of subjects with low risk of extubation failure occurs in $10 \%$ to $15 \%$ of cases, increasing to $25 \%$ to $30 \%$ in subjects with high risk [4]. One possible reason for these false-positive results is that the SBT does not demonstrate whether subjects can protect their lower airways after extubation. In this way, considering that ineffective cough and secretion retention can determine or contribute to extubation failure [5], measuring cough peak flow before extubation has been proposed as an alternative to evaluating a subject's ability in clearing secretions. The cough peak flow can be obtained voluntarily, when the patient is verbally asked to cough as hard as possible or involuntary when the reflex cough is inducted by saline instillation or with a catheter. Many authors demonstrated that voluntary [6-8] or involuntary [9-11] CPF has high accuracy in predicting the extubation outcome; however, only Duan et al. [10] compared these two forms of stimulation regarding their predictive power. They found that, in a sample of cooperative subjects, voluntary is better than involuntary cough in predicting the extubation outcome. Voluntary CPF can result in underestimated values because it depends on the motivation of the subjects. On the other hand, the magnitude of the reflex stimulation highly influences the involuntary cough response and, therefore, the resulting CPF. It is currently unknown which type of cough (voluntary or involuntary) would be more accurate in assessing readiness for extubation. Moreover, we found no studies assessing the predictive power of $\mathrm{CPF}$ measured after extubation. Therefore, we aimed to investigate if verbalvoluntary cough stimulation, cough stimulation with saline instillation, and mechanical cough stimulation by catheter are equivalent in predicting the extubation outcome. Additionally, we also examined if CPF measured after endotracheal tube removal can predict the extubation outcome.

\section{METHODS}

Study design and sample characteristics

This was a prediction, observational, prospective study, including a convenience sample of mechanically ventilated subjects. Data were collected at the Dr. Mozart Teixeira Hospital (HPS) in the city of Juiz de Fora, MG, in the Emergency Room and Intensive Care Unit. Any subjects under mechanical ventilation for more than $24 \mathrm{~h}$, considered ready for weaning defined by improvements in underlying medical conditions, withdrawal or low-dose of pressors or inotropes, $\mathrm{PaO}_{2} \geq 60 \mathrm{mmHg}$ with $\mathrm{FIO}_{2} \leq 0.5, \mathrm{PEEP} \leq 5 \mathrm{cmH}_{2} \mathrm{O}$, temperature $\leq 38^{\circ} \mathrm{C}$, systolic blood pressure between 90 and $180 \mathrm{mmHg}$, heart rate < 140 beats/min, and hemoglobin $>7 \mathrm{~g} / \mathrm{dL}$, and who succeeded in the SBT were included. Subjects who

\footnotetext{
${ }^{1}$ Rehabilitation Sciences Post-Graduation Program, Augusto Motta University, Rio de Janeiro, Brazil

${ }^{2}$ Post-Graduation Program in Medical Sciences, School of Medical Sciences, State University of Rio de Janeiro, Rio de Janeiro, Brazil

${ }^{3}$ Physical Therapy Department, Medical School, Federal University of Rio de Janeiro, Rio de Janeiro, Brazil

Correspondence: Fernando Guimarães, Serviço de Fisioterapia - Hospital Universitário Clementino Fraga Filho - UFRJ, R. Prof. Rodolpho Paulo Rocco, 255 -

Cidade Universitária da Universidade Federal do Rio de Janeiro, Rio de Janeiro, RJ 21941-590. Tel: +55 21 99124-3760, E-mail: fguimaufri@yahoo.com.br
}

Published online at https://www.cjrt.ca on 20 November 2020 
were unable to respond to the coughing command or who had postextubation laryngospasm were excluded from the study. The study was approved by the ethics committee of the University Augusto Motta (CAAE: 54341716.4.0000.5235), and informed consent was obtained from subjects or their relatives. The sample size was estimated according to the method described by Lu et al. [12]. Considering the results of Duan et al. [10], type I error of $5 \%$, type II error of $20 \%$, expected mean of differences of $7.8 \mathrm{~L} / \mathrm{min}$, and expected standard deviation of differences of $40 \mathrm{~L} / \mathrm{min}$, a required sample size of 75 subjects was necessary for BlandAltman agreement assessment.

\section{Procedures and outcome measures}

Weaning readiness was assessed daily by the attending physician and a physical therapist. The maximal inspiratory and expiratory pressures (MIP and MEP) were measured with a previously calibrated manovacuometer (M120 healthcare; São Paulo, Brazil) coupled to a unidirectional valve through a T-piece. The patients were disconnected from the ventilator, and the T-piece was connected to the endotracheal tube. The recordings were done with the patient in a $45^{\circ}$ Fowler position with an occlusion time of $30 \mathrm{~s}$. Two measurements were performed with a 2-min interval between them, considering the highest value as representative. A ventilometer (FERRARIS Wright ${ }^{\circledR}$ MK 8. Louisville, USA) was used to record the minute ventilation and tidal volume in spontaneous ventilation, allowing for the calculation of the rapid shallow breathing index (RSBI) before extubation.

Subjects who met the weaning criteria underwent an SBT via a T-piece or with pressure support of $7 \mathrm{cmH}_{2} \mathrm{O}$ for $30 \mathrm{~min}$. The subjects were considered unsuccessful in the SBT if they presented any sign of poor tolerance, such as a respiratory rate $\geq 35$ breaths / min, arterial oxygen saturation $<90 \%$ (while breathing $50 \%$ of oxygen), heart rate $>140$ beats $/ \mathrm{min}$, systolic blood pressure $>180 \mathrm{mmHg}$ or $<90 \mathrm{mmHg}$, agitation, diaphoresis, or anxiety. When subjects passed the SBT, before extubation, we measured the CPF using three methods: involuntary CPF with saline instillation (S_CPF), involuntary $\mathrm{CPF}$ with catheter stimulation $\left(\mathrm{C}_{-} \mathrm{CPF}\right)$, and voluntary $\mathrm{CPF}$ (V_CPF). The subjects were suctioned, oxygenated with $100 \%$ oxygen for 2 min, and positioned at $45^{\circ}$, and a portable peak flow meter ( $\mathrm{PiKo}-1$ spirometer, nSpire Health, Hertford, UK) was used for CPF measurements. The peak flow meter was connected to the endotracheal tube by a filter and via a T-piece with a one-way valve to enable free inhalation and exhalation. A closed suction catheter was connected to this system, allowing for the saline instillation, and the subjects were under continuous vital signs monitoring throughout the procedure. Two measures were used for every CPF method with a minimum 3-min interval, and the highest value was computed. The order of CPF recordings was determined by block randomization, and a recovery period of $15 \mathrm{~min}$ was allowed between the different CPF methods. Additionally, we measured the voluntary cough peak flow $20 \mathrm{~min}$ after the extubation (PE_CPF) using a mask connected to the spirometer.
These recordings were done twice and provided that the patient was stable and without signs of respiratory failure. To measure V_CPF and PE_CPF, the subjects were instructed to breathe in deeply, and then cough as hard as possible (e.g., the clinician would state "when I say go, take a deep breath and cough as hard as possible"). For S_CPF, the stimulation consisted of $3 \mathrm{~mL}$ of normal saline instilled by the closed suction catheter port, and, for C_CPF, a lubrified suctioning catheter $(8 \mathrm{~F})$ was inserted through the patient's nasal pathway, reaching the reflex area above the glottis [11]. Extubation was considered unsuccessful when reintubation was required in less than $48 \mathrm{~h}$. The criteria for reintubation were as follows: RR $>30 \mathrm{bpm}$, $\mathrm{SpO}_{2}<90 \%$ with $\mathrm{FiO}_{2} \geq 0.5, \mathrm{pH}<7.3$, persistent tachycardia, diminished consciousness, diaphoresis, clinical signs of respiratory muscle fatigue, and persistent activation of accessory respiratory muscles [13].

\section{Statistical analysis}

The patients' characteristics were expressed as mean and standard deviation, as well as absolute and relative frequency. The data distribution was tested using the Kolmogorov-Smirnov test. Student's t-test for independent samples and the chi-square test were used to assess differences between groups (success and failure in extubation). Effect size was evaluated by Cohen's d (effect sizes above 0.8 and 1.3 are considered large and very large, respectively) [14]. A receiving operating characteristic (ROC) curve assessed the sensitivity and specificity of diagnostic accuracy of the CPF methods (S_CPF, V_CPF, PE_CPF, and C_CPF), RSBI, and MIP in relation to the extubation outcome. The method of Hanley and McNeil [15] was used to compare the areas under the ROC curves, and the optimal cutoff point was chosen using the maximum Youden index [16]. Additionally, S_CPF, V_CPF, and C_CPF were compared using the Altman-Bland plots, and the Intraclass Correlation Coefficient (ICC) was calculated to access reliability. Analyses were done using MedCalc Statistical Software version 17.2 (MedCalc Software bvba, Ostend, Belgium) and IBM SPSS version 21.0 (IBM Corp., Armonk, NY). The value of $\alpha \leq 0.05$ was adopted for statistical significance.

\section{RESULTS}

One hundred and thirteen patients were assessed for weaning. From these, 31 did not pass in SBT, and one presented laryngospasm postextubation. Thus, the study included 81 subjects who were extubated between May 2016 and February 2017. The general characteristics of the subjects are presented in Table 1 . The mean age of the subjects was $46 \pm$ 20 years, ranging from 14 to 94 years. There was a predominance of male subjects $(78 \%)$. The most common clinical diagnoses were polytrauma $(n=13,16 \%)$, traumatic brain injury $(n=13,16 \%)$, firearm injuries $(n=$ $8,10 \%)$, and postoperative laparotomy $(n=7,9 \%)$. Of the reintubated subjects, $48.5 \%$ were due to acute respiratory failure, $24.2 \%$ due to lowering of consciousness level, $9.1 \%$ due to agitation, and $18.2 \%$ due to other causes (e.g. bronchospasm, hypotension, secretion retention, etc.).

Table 1

Patients' characteristics

\begin{tabular}{|c|c|c|c|c|c|}
\hline Variables & Total $(n=81)$ & $\begin{array}{l}\text { Extubation success } \\
\qquad(n=48)\end{array}$ & $\begin{array}{l}\text { Extubation failure } \\
\qquad(n=33)\end{array}$ & $P$ & Effect size \\
\hline Age, years & $46.0 \pm 20.0$ & $37.5 \pm 16.7$ & $58.4 \pm 18.0$ & $<0.0001$ & 1.16 \\
\hline Sex (male, \%) & $77.8 \%$ & $81.3 \%$ & $72.7 \%$ & 0.36 & 0.10 \\
\hline Duration of MV, days & $3.6 \pm 2.6$ & $3.2 \pm 2.1$ & $4.2 \pm 3.0$ & 0.11 & 0.33 \\
\hline APACHE II & $8.0 \pm 0.3$ & $8.4 \pm 5.0$ & $21.7 \pm 7.2$ & $<0.0001$ & 1.85 \\
\hline $\operatorname{MIP}\left(\mathrm{cmH}_{2} \mathrm{O}\right)$ & $44.7 \pm 11.0$ & $50.5 \pm 9.7$ & $36.4 \pm 6.5$ & $<0.0001$ & 2.17 \\
\hline $\operatorname{MEP}\left(\mathrm{cmH}_{2} \mathrm{O}\right)$ & $38.5 \pm 9.2$ & $43.1 \pm 7.8$ & $31.7 \pm 6.6$ & $<0.0001$ & 1.73 \\
\hline RSBI (cycles/min/L) & $48.9 \pm 10.2$ & $44.8 \pm 8.5$ & $54.9 \pm 9.6$ & $<0.0001$ & 1.05 \\
\hline S_CPF (L/min) & $68.7 \pm 17.3$ & $79.2 \pm 13.0$ & $53.5 \pm 9.7$ & $<0.0001$ & 2.65 \\
\hline C_CPF (L/min) & $61.1 \pm 15.5$ & $69.5 \pm 13.4$ & $49.0 \pm 9.1$ & $<0.0001$ & 2.25 \\
\hline V_CPF (L/min) & $55.5 \pm 15.3$ & $63.3 \pm 14.4$ & $44.1 \pm 7.6$ & $<0.0001$ & 2.53 \\
\hline PE_CPF (L/min) & $80.7 \pm 24.8$ & $96.2 \pm 18.2$ & $58.2 \pm 12.8$ & $<0.0001$ & 2.97 \\
\hline
\end{tabular}

Note: Values are mean \pm SD. APACHE II, Acute Physiology and Chronic Health Disease Classification System II; MIP, maximal inspiratory pressure; MEP, maximal expiratory pressure; RSBI, Rapid Shallow Breathing Index; S_CPF, cough peak flow recorded using stimulation with saline instillation before extubation; C_CPF, cough peak flow recorded using catheter stimulation before extubation; V_CPF, voluntary cough peak flow before extubation; PE_CPF, voluntary cough peak flow recorded $20 \mathrm{~min}$ after the extubation. 


\section{TABLE 2}

Predictive accuracy of cough peak flow measurements, maximal inspiratory pressure, and rapid shallow breathing index

\begin{tabular}{|c|c|c|c|c|c|}
\hline Variable & Cutoff & Sensitivity (\%) & Specificity (\%) & AUC (CI 95\%) & $P$ \\
\hline S_CPF (L/min) & $>60$ & 91.7 & 87.9 & $0.93(0.85-0.97)$ & $<0.0001$ \\
\hline C_CPF (L/min) & $>55$ & 85.4 & 90.9 & $0.90(0.81-0.95)$ & $<0.0001$ \\
\hline V_CPF (L/min) & $>45$ & 93.7 & 69.7 & $0.89(0.80-0.95)$ & $<0.0001$ \\
\hline$P \bar{E} \_C P F(L / m i n)$ & $>75$ & 85.4 & 97.0 & $0.95(0.88-0.98)$ & $<0.0001$ \\
\hline $\operatorname{MIP}\left(\mathrm{cmH}_{2} \mathrm{O}\right)$ & $>45$ & 62.5 & 100.0 & $0.87(0.77-0.93)$ & $<0.0001$ \\
\hline RSBI (cycles/min/L) & $\leq 50$ & 77.1 & 69.7 & $0.79(0.69-0.87)$ & $<0.0001$ \\
\hline
\end{tabular}

Note: S_CPF, cough peak flow recorded using stimulation with saline instillation before extubation; C_CPF, cough peak flow recorded using catheter stimulation before extubation; V_CPF, voluntary cough peak flow before extubation; PE_CPF, voluntary cough peak flow recorded 20 min after the extubation; MIP, maximal inspiratory pressure; RSBI, Rapid Shallow Breathing Index; AUC, area under the ROC curve.

\section{FIGURE 1}

Comparison between receiver operating characteristics curves of cough peak flow measures to predict the extubation outcome. S_CPF, cough peak flow stimulated with $3 \mathrm{~mL}$ of saline instillation, C_CPF = cough peak flow stimulated with catheter, V_CPF, voluntary (coached) cough peak flow.

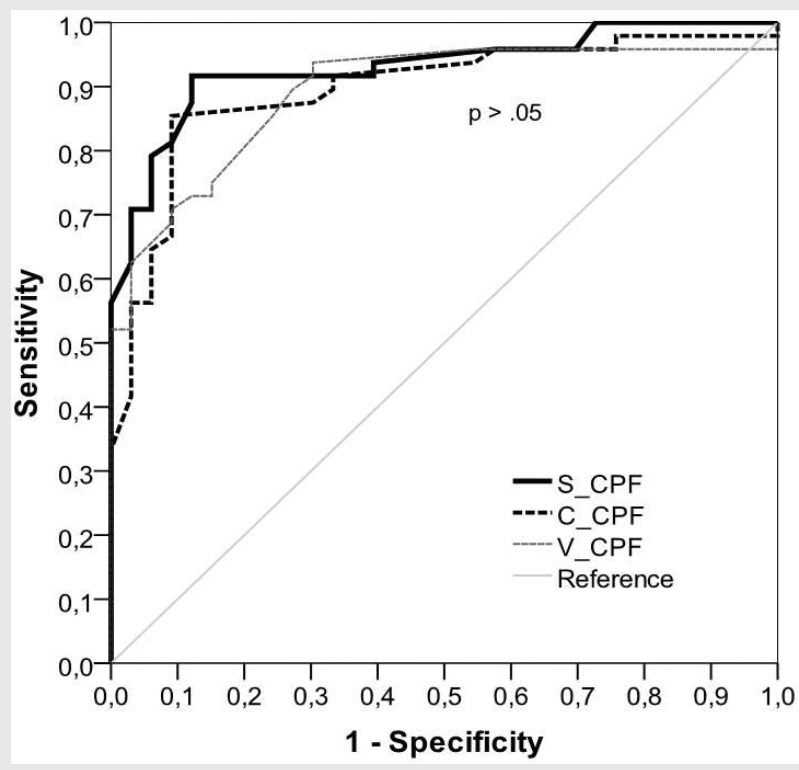

Subjects who succeeded in extubation were younger and had lower scores on APACHE II and higher values of MIP, MEP, RSBI, and RR (Table 1). There was no statistically significant difference between the diagnostic accuracy of the CPF methods (S_CPF vs. C_CPF: $p=0.13$; S_CPF vs. V_CPF: $p=0.14$; C_CPF vs. V_CPF: $p=0.84)$. There was also no statistically significant difference in the accuracy when comparing RSBI and MIP $(p=0.20)$. The predictive power of CPF (S_CPF, V_CPF, PE_CPF, and C_CPF), RBSI, and MIP in relation to the successful outcome of the extubation is presented in Table 2 and Figures 1 and 2. Bland-Altman plots (Figures 3-5) showed agreement between the CPF methods, with a relatively small systematic bias. The intra-rater reliability considering the three methods was excellent $(\mathrm{ICC}=0.914)$.

\section{DISCUSSION}

The findings of this study are in agreement with those of previous authors who demonstrated the strong power of CPF in predicting the extubation outcome in subjects who passed an SBT [6, 7, 9-11, 17]. Cough peak flow was significantly lower in patients with failed extubation $(P<0.0001$; effect size $>2.2$ in all cough stimulation methods). To identify the best way to obtain the CPF in intubated subjects, we compared three forms of cough stimulation: with instillation of saline, with mechanical stimulation by catheter, and by means of verbal stimulation. There was no difference between these methods with respect to the diagnostic accuracy; however, the saline stimulation presented the best combination of sensitivity and specificity. Moreover, a measure of CPF done after extubation also had high accuracy. The Bland-Altman analysis showed that the three methods are interchangeable, as there were no proportional or systematic errors, and the percentage of points outside the agreement limits was very low: $\mathrm{S}_{-}$ CPF versus C_CPF (7.4\%), S_CPF versus V_CPF (4.9\%) and C_CPF versus $V_{-}$CPF (7.4\%). It is likely that in the real clinical context these percentages are even lower, since a smaller number of measures are performed, thus avoiding the occurrence of fatigue and progressive reduction of $\mathrm{CPF}$ in some patients. Moreover, the agreement between methods was corroborated by the ICC (0.914).

As in our study, Duan et al. [10] compared the predictive power of CPF using voluntary and involuntary (reflex) maneuvers. They observed that voluntary was better than involuntary CPF in predicting the 


\section{FIGURE 2}

Receiver operating characteristics curve of cough peak flow measure to predict the extubation outcome. The measure was performed after extubation using a spirometer connected to a facemask.

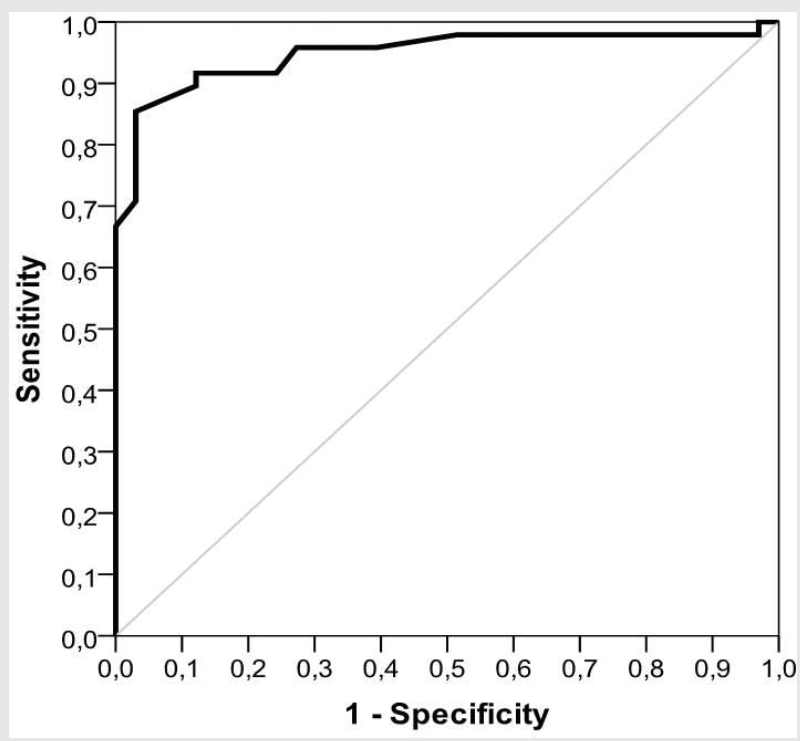

\section{FIGURE 3}

Bland-Altman plot showing the bias and the limits of agreement (bias $\pm 1.96 \mathrm{SD}$ ); the horizontal axis shows the mean determinations using the 2 methods and the vertical axis shows the difference between the methods. S_CPF, cough peak flow stimulated with $3 \mathrm{~mL}$ of saline instillation, C_CPF = cough peak flow stimulated with catheter.

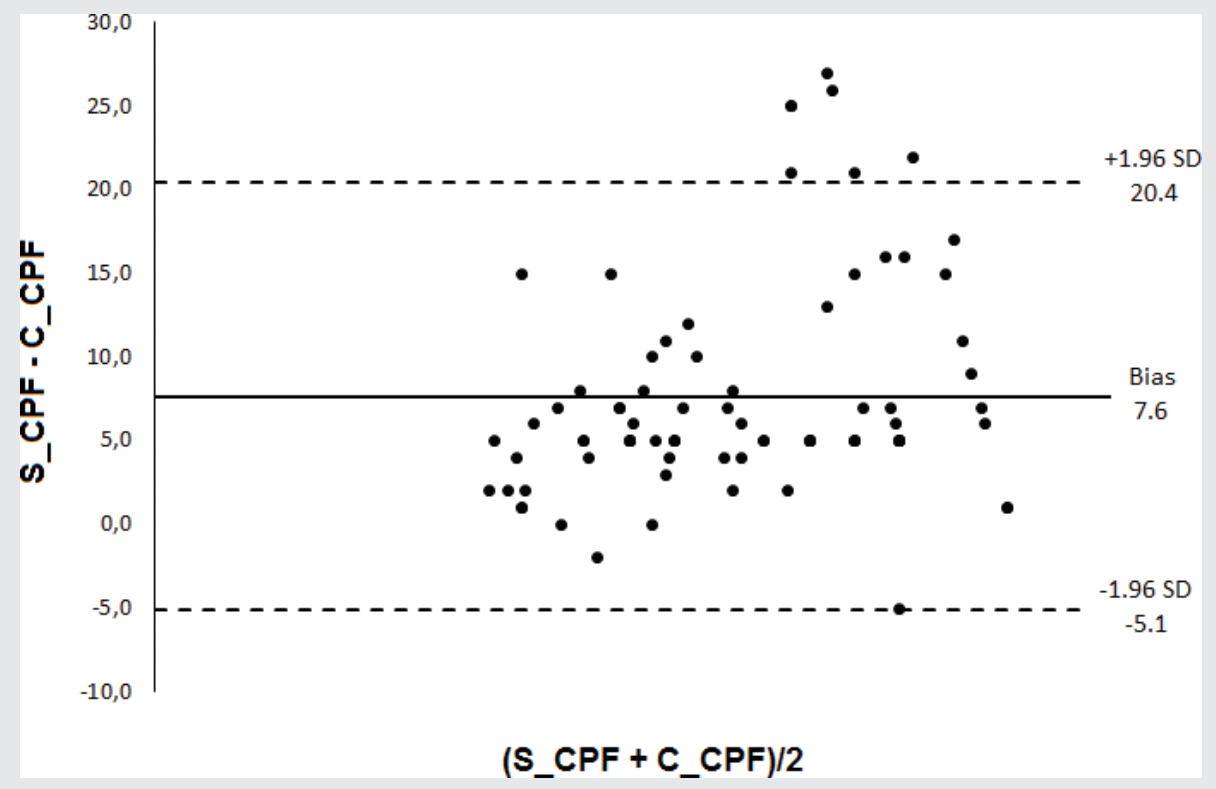




\section{FIGURE 4}

Bland-Altman plot showing the bias and the limits of agreement (bias $\pm 1.96 \mathrm{SD}$ ); the horizontal axis shows the mean determinations using the 2 methods and the vertical axis shows the difference between the methods. S_CPF, cough peak flow stimulated with $3 \mathrm{~mL}$ of saline instillation, V_CPF = voluntary (coached) cough peak flow.

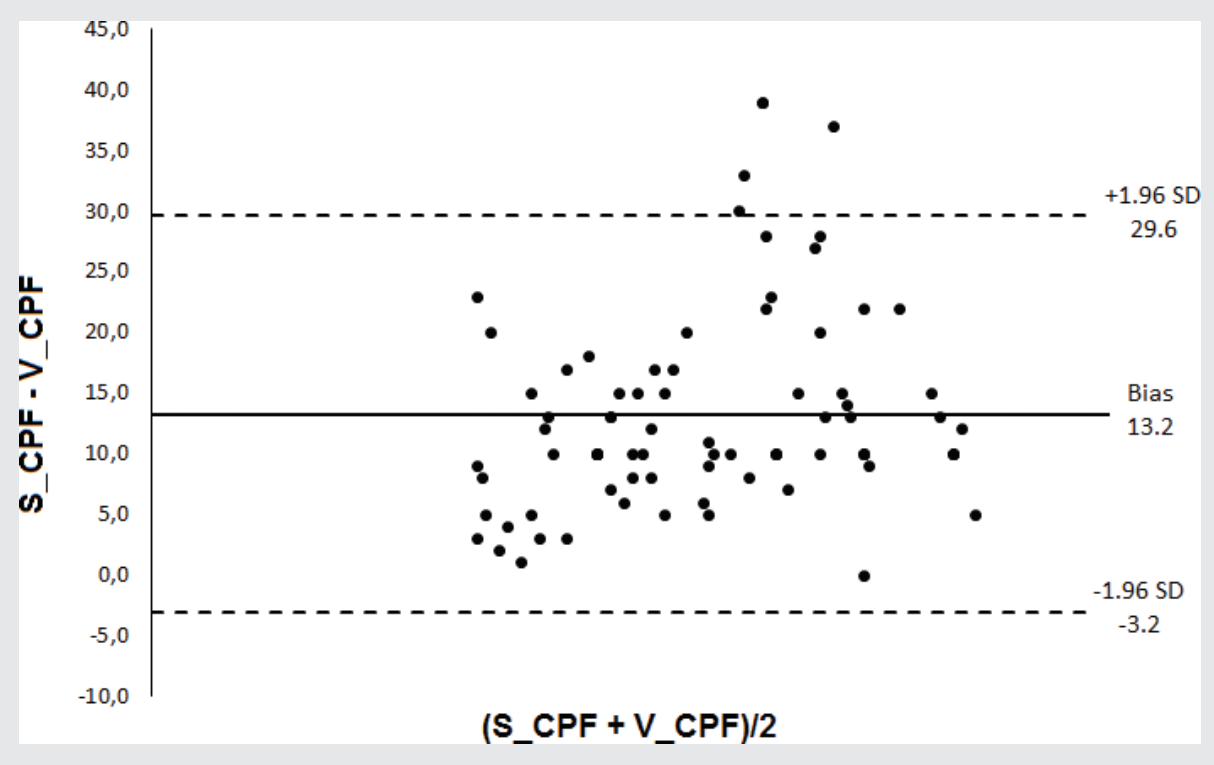

\section{FIGURE 5}

Bland-Altman plot showing the bias and the limits of agreement (bias $\pm 1.96 \mathrm{SD}$ ); the horizontal axis shows the mean determinations using the 2 methods and the vertical axis shows the difference between the methods. C_CPF = cough peak flow stimulated with catheter, V_CPF, voluntary (coached) cough peak flow.

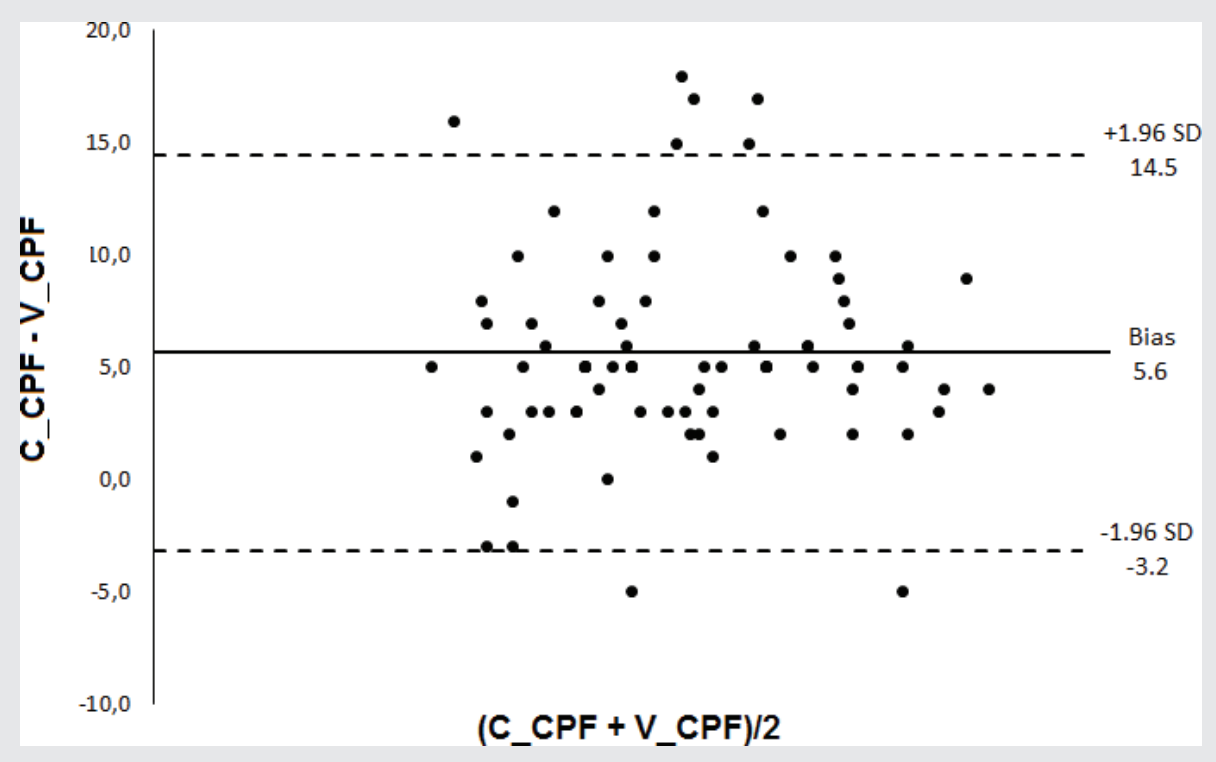


extubation outcome in a sample of cooperative subjects. Differently, in our study, we found no significant accuracy differences between voluntary (coached) and involuntary (saline) maneuvers. Because the magnitude of the cough response is stimulus dependent, it is likely that the higher volume of saline used in our study $(3 \mathrm{~mL}$ vs. $2 \mathrm{~mL})$ determined these differences. The fact that the S_CPF observed in our study was higher than the V_CPF seems to corroborate the hypothesis of understimulation in Duan et al. [10] because they found lower V_CPF values. In this way, we can assume that the reflex stimulation would be more reliable than the verbal one because it does not depend on the patient's motivation. However, to be reliable, one must ensure that the magnitude of the reflex stimulus is sufficient to elicit a maximal response.

Likewise, we tested the involuntary cough using the catheter stimulation and found no accuracy differences in relation to the other methods, confirming its utility for CPF measurement in intubated subjects. In this regard, we observed an AUC of 0.9, which is higher than that from Kutchak et al. [11] (0.81) who assessed a sample of subjects with varied neurological conditions, which might have presented with other extubation failure determinants, such as dysphagia and lowered level of consciousness. The fact that only $31 \%$ of their subjects required reintubation because of retained secretions, in contrast with $62 \%$ of subjects who failed in extubation due to incapacity to protect their airways, corroborates this hypothesis.

Regarding the cutoff values for predicting the extubation outcome, our S_CPF and C_CPF results (60 and $55 \mathrm{~L} / \mathrm{min}$, respectively) are similar to those previously reported $[6,8-10]$. On the other hand, we found a cutoff of only $45 \mathrm{~L} / \mathrm{min}$ for V_CPF, which is lower than that observed by other authors when using verbal stimulation. This is an expected result because in our study the values of CPF obtained voluntarily were lower than those obtained using the reflex stimulation methods. A reduced cutoff of $35 \mathrm{~L} /$ min when assessing V_CPF was also found in Beuret et al. [7]. Although methodological differences between studies might have influenced the optimal cutoff value, these results suggest that voluntary coughing may underestimate the true CPF of subjects. Thus, based on this premise, V_CPF may be the first choice for evaluating the CPF in cooperative subjects because it is less stressful for the patient. However, to avoid underestimating results, a cutoff of $55-60 \mathrm{~L} / \mathrm{min}$ should be considered a clinical reference, as reported in the literature $[6,8-10]$ and this study's results. If values below $55-60 \mathrm{~L} / \mathrm{min}$ are measured with voluntary cough, only then a counterproof measurement using reflex stimulation might be performed. This stimulation can be done using at least $3 \mathrm{~mL}$ of saline instillation; however, this procedure might dislodge bacteria-laden biofilm from the endotracheal tube, potentially causing more pulmonary infections [18]. Alternatively, the catheter stimulation is not associated with any potential risk of bacterial translocation and has good predictive power, as demonstrated by Kutchak et al. [11] (accuracy of 0.8) and in our study $(\mathrm{AUC}=0.90)$.

In addition to the pre-extubation measures, we evaluated the predictive power of CPF after endotracheal tube removal. Obviously, this measure does not help in the extubation decision-making process; however, because its accuracy was high, it seems to be a promising way to evaluate subjects without artificial airway regarding the risk of potential intubation. It is likely that intubated subjects with $\mathrm{CPF}<55-60 \mathrm{~L} / \mathrm{min}$ or extubated with $\mathrm{CPF}<75 \mathrm{~L} / \mathrm{min}$ can benefit from cough-enhancement strategies in the post-extubation period. Although there is a clear theoretical rationale in this regard, a recent meta-analysis concluded that the effect of cough augmentation techniques to facilitate extubation and prevent postextubation respiratory failure is unclear [19]. A possible bias in the clinical trials about the use of cough augmentation techniques, such as mechanical insufflation-exsufflation, is the inclusion of subjects who did not present with weak cough strength and, therefore, do not benefit from this kind of therapy. This is corroborated by Duan et al. [20], who found that postextubation noninvasive ventilation (NIV) reduced the intubation rate and post-extubation 90-day mortality in subjects with $\mathrm{CPF}<70 \mathrm{~L} / \mathrm{min}$, but without any benefit in subjects with strong cough (>70 L/min) [20]. If these authors found this positive result with NIV, which is not intended to increase cough efficiency, it is possible that cough-augmenting techniques could prove to be even more effective in avoiding intubation in subjects with reduced CPF in future studies.

Like previous authors, we found significant differences (with large or very large effect size) between subjects who succeeded and failed in the extubation in the parameters of age, severity, RSBI, MIP, and MEP $[9,11]$. Among these, RSBI and MIP were tested regarding their predictive power, showing good accuracy. Despite this positive result regarding RSBI, researchers have found important differences in its accuracy depending on the application method and target population. Even so, it remains a practical method of predicting the patient's integrated capacity to sustain spontaneous ventilation. However, to predict subjects' ability to protect their airways and eliminate secretions in the post-extubation period, the assessment of other parameters such as cough adequacy and the amount of secretions is necessary [21]. CPF is a promising tool because it can predict the outcome of extubation in subjects who have already been successful in the SBT. Even if the CPF test results are not decisive enough to extubate, it may be used in the future to help in the risk stratification, indicating which subjects might require further monitoring care and prophylactic procedures in the post-extubation period, such as NIV or coughing augmentation techniques [22]. Alternatively, the performance of weaning predictive indexes in patients with neuromuscular diseases has been found to be poor [13]. As we investigated a general sample of mechanically ventilated patients, future studies should address the predictive power of $\mathrm{CPF}$ in patients with neurologic/neuromuscular conditions.

\section{CONCLUSION}

In conclusion, in mechanically ventilated and cooperative subjects, there is no difference in the accuracy of the CPF measured voluntarily, with stimulation using $3 \mathrm{~mL}$ of saline, or by catheter stimulation in predicting the reintubation. CPF recording after the endotracheal tube removal also demonstrated high accuracy to predict the extubation outcome.

\section{DISCLOSURES}

\section{Competing interests}

All authors have completed the ICMJE uniform disclosure form at www. icmje.org/coi_disclosure.pdf and declare: no financial relationships with any organizations that might have an interest in the submitted work in the previous 3 years; no other relationships or activities that could appear to have influenced the submitted work.

\section{Ethical approval}

The study was approved by the ethics committee of the University Augusto Motta (CAAE: 54341716.4.0000.5235), and informed consent was obtained from subjects or their relatives.

\section{REFERENCES}

1. MacIntyre NR, Cook DJ, Ely EW Jr, et al. Evidence-based guid elines for weaning and discontinuing ventilatory support: a collective task force facilitated by the American College of Chest Physicians; the American Association for Respiratory Care; and the American College of Critical Care Medicine. Chest 2001;120(6 Suppl):375S-95S. doi: 10.1378/ chest.120.6_suppl.375S.

2. Jiang C, Esquinas A, Mina B. Evaluation of cough peak expiratory flow as a predictor of successful mechanical ventilation discontinuation: a narrative review of the literature. J Intensive Care 2017;5(1):33. doi: 10.1186/s40560-017-0229-9.

3. Boles JM, Bion J, Connors A, Herridge M, Marsh B, Melot C, et al. Weaning from mechanical ventilation. Eur Respir J 2007;29(5):1033-56. doi: 10.1183/09031936.00010206.

4. Thille AW, Richard JCM, Brochard L. The decision to extubate in the intensive care unit. Am J Respir Crit Care Med 2013;187(12):1294-302. doi: $10.1164 / \mathrm{rccm} .201208-1523 \mathrm{CI}$.

5. Perren A, Brochard L. Managing the apparent and hidden difficulties of weaning from mechanical ventilation. Intensive Care Med 2013;39(11):1885-95. doi: 10.1007/s00134-013-3014-9.

6. Smina M, Salam A, Khamiees M, Gada P, Amoateng-Adjepong Y, Manthous CA. Cough peak flows and extubation outcomes. Chest 2003;124(1):262-8. doi: 10.1378/chest.124.1.262. 
7. Beuret P, Roux C, Auclair A, Nourdine K, Kaaki M, Carton MJ. Interest of an objective evaluation of cough during weaning from mechanical ventilation. Intensive Care Med 2009;35(6):1090-3.

8. Smailes ST, McVicar AJ, Martin R. Cough strength, secretions and extubation outcome in burn subjects who have passed a spontaneous breathing trial. Burns 2013;39(2):236-42. doi: 10.1016/j.burns.2012. 09.028.

9. Su WL, Chen YH, Chen CW, et al. Involuntarycough strength and extubation outcomes for subjects in an ICU. Chest 2010;137(4):777-82. doi: 10.1378/chest.07-2808.

10. Duan J, Liu J, Xiao M, Yang X, Wu J, Zhou L. Voluntary is better than involuntary cough peak flow for predicting re-intubation after scheduled extubation in cooperative subjects. Respir Care 2014;59(11):1643-51. doi: $10.4187 /$ respcare. 03045 .

11. Kutchak FM, Debesaitys AM, Rieder Mde M, et al. Reflex cough PEF as a predictor of successful extubation in neurological subjects. J Bras Pneumol 2015;41(4):358-64. doi: 10.1590/S1806-3713201500 0004453.

12. Lu MJ, Zhong WH, Liu YX, Miao HZ, Li YC, Ji MH. Sample size for assessing agreement between two methods of measurement by BlandAltman Method. Int J Biostat 20161;12(2):/j/ijb.2016.12.issue-2/ijb2015-0039/ijb-2015-0039.xml. doi: 10.1515/ijb-2015-0039.

13. de Souza LC, Guimarães FS, Lugon JR. The timed inspiratory effort: a promising index of mechanical ventilation weaning for patients with neurologic or neuromuscular diseases. Respir Care 2015;60(2):231-8. doi: $10.4187 /$ respcare.03393.
14. Sullivan GM, Feinn R. Using effect size-or why the P value is not enough. J Grad Med Educ 2012;4(3):279-82. doi: 10.4300/JGME-D-12-00156.1.

15. Hanley JA, McNeil BJ. A method of comparing the areas under receiver operating characteristic curves derived from the same cases. Radiology 1983;148(3):839-43.

16. Youden WJ. Index for rating diagnostic tests. Cancer 1950;3(1):32-5. doi: 10.1002/1097-0142(1950)3:1<32::AID-CNCR2820030106>3.0.CO;2-3.

17. Winck JC, LeBlanc C, Soto JL, Plano F. The value of cough peak flow measurements in the assessment of extubation or decannulation readiness. Rev Port Pneumol 2015;21(2):94-8. doi: 10.1016/j. rppnen.2014.12.002.

18. Branson RD. Secretion management in the mechanically ventilated patient. Respir Care 2007;52(10):1328-42.

19. Rose L, Adhikari NK, Leasa D, Fergusson DA, McKim D. Cough augmentation techniques for extubation or weaning critically ill subjects from mechanical ventilation. Cochrane Database Syst Rev 2017 Jan 11;1:CD011833. doi: 10.1002/14651858.CD011833.pub2.

20. Duan J, Han X, Huang S, Bai L. Noninvasive ventilation for avoidance of reintubation in subjects with various cough strength. Crit Care 2016;20(1):316-22. doi: 10.1186/s13054-016-1493-0.

21. Karthika M, Al Enezi F, Pillai L, Arabi Y. Rapid shallow breathing index. Ann Thorac Med 2016;11(3):167. doi: 10.4103/1817-1737.176876.

22. Terzi N, Guerin C, Gonçalves MR. What's new in management and clearing of airway secretions in ICU subjects? It is time to focus on cough augmentation. Intensive Care Med 2019;45(6):865-68. doi: 10.1007/ s00134-018-5484-2. 